\title{
Management of Upper Airway Bleeding in Patients Hos- pitalized for SARS-COV2
}

\author{
Hernandez Mundo A, Mancilla Mejia F, Serrano Salinas L, Dirzo Cuevas S, Mendez Saucedo L, Garcia Rodriguez E, \\ Toledo Perez A, Sanchez Mendoza J, Mora Perez J* \\ Department of Otorhinolaryngology Head and Neck Surgery. Centro Médico Naval. Mexico.
}

\begin{abstract}
Spontaneous epistaxis in patients with COVID-19 can represent a clinical challenge with respect to both the risk of contamination and the treatment options. Epistaxis (nosebleed) is a common condition overall but is relatively rare in adults and older patients. We herein present the data of 25 patients with COVID-19 who developed spontaneous epistaxis while hospitalized at Centro Médico Naval from March 2020 to April 2021. All patients received low-molecular-weight heparin during their hospital stay and required supplementary oxygen therapy either by a nasal cannula, continuous positive airway pressure or ventilatory mechanical support.
\end{abstract}

In total, 12100 patients with laboratory confirmed SARS-CoV-2 infection were admitted to our hospital unit from 1 March to 31 April 2021 . We only found 25 patients with epistaxis, All patients underwent anterior rhinoscopy.

In all patients, the bleeding site was identified, controlled with cauterization with silver nitrate and absorbable hemostatic material. In patients with posterior epistaxis, the posterior packing was removed after 5 days. Nasal lubricants were prescribed in all patients after bleeding was controlled. All patients required suspension of anticoagulation after the bleeding event for 48 hours.

Posterior, anterior and oropharyngeal epistaxis occurred in both sexes, in patients with comorbidities (Diabetes mellitus, hypertension, obesity) without statistical difference. In those patients who were on ventilatory mechanical support, they presented the same risk of epistaxis, however, with a small tendency to present greater anterior epistaxis, in the same way, those patients who presented anterior epistaxis had higher mortality.

Keywords: COVID, Patients, Hypertension, Obesity

\section{Introduction}

Upper airway bleeding is a potential emergency that can end fatally if not controlled quickly and accurately. Since it affects the hemodynamic balance, ventilation and makes intubation difficult if it is necessary to secure the airway. ${ }^{1}$ The SARS-CoV-2 infection includes among the clinical manifestations thrombocytopenia or thromboembolic complications, which necessitate anticoagulation or the use of vasopressors. Manipulation of the airway is necessary from the onset of suspicion of the disease; the diagnosis requires taking a nasopharyngeal swab for RT-PCR, a procedure that is associated between 5-10\% with epistaxis. ${ }^{1}$
The supply of supplemental oxygen is necessary in patients who develop pneumonia 2; the use of conventional nasal tips, high-flow nasal tips and positive pressure devices are factors that can be associated with nasal bleeding; In addition, in critically ill patients with intubation, the aspiration of secretions in the oral cavity, oropharynx and hypopharynx can cause trauma to the mucosa and cause bleeding. ${ }^{2-4}$ The most frequent site of bleeding in the upper airway is located in the nose, followed by the oral cavity and pharyngeal regions. Kristensen, 2020 Epistaxis is one of the most common emergencies in Otolaryngology (ENT), it has an incidence of $13.9 \%$ in care hospitable. Kristensen, 2020 Posterior hemorrhage is the

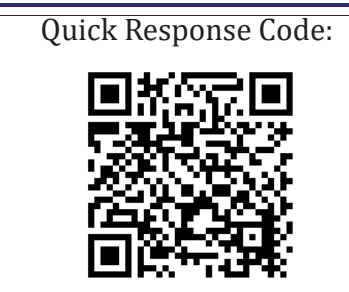

Copyright (C) All rights are reserved by Josue Mora Perez

\footnotetext{
*Corresponding author: Josué Mora Pérez. Av. Heríca escuela Naval Militar 745. Presidentes Ejidales 1 era Sec. Coyoacán. CP 04470. Mexico

Received: 15 June, 2021

Published: 14 July, 2021

Citation: Hernandez MA, Mancilla MF, Serrano SL, Dirzo Cs, Mendez SL, et al. Management of Upper Airway Bleeding in Patients Hospitalized for SARS-COV2. SOJ Complement Emerg Med. 2021;1(2):1-4. DOI: 10.53902/SOJCEM.2021.01.000509
} 
most serious presentation and is generated mainly from branches of the sphenopalatine artery. ${ }^{5}$

Mortality associated with epistaxis is $3.4 \%$. Death is not considered to be a direct result of the bleeding. However, blood loss contributes to increased morbidity. It can be a direct cause of death from hemorrhagic shock or from aspiration of blood and clots into the lungs. ${ }^{6}$

\section{Methods}

We describe a series of cases of hospitalized patients in the
COVID-19 area of the Centro Médico Naval, with a diagnosis confirmed by RT-PCR and who developed spontaneous bleeding from the upper respiratory tract and required ENT evaluation for management and control. This research was conducted in accordance with the ethical principles originating in the Declaration of Helsin$\mathrm{ki}$, and written informed consent was obtained from all patients. All personnel used personal protection measures (Figure 1) and international recommendations to limit exposure to aerosols during the management of bleeding in the upper respiratory tract.7, ${ }^{7,8}$ examination was carried out with a headlamp. In no case was the use of endoscopes required to control bleeding.
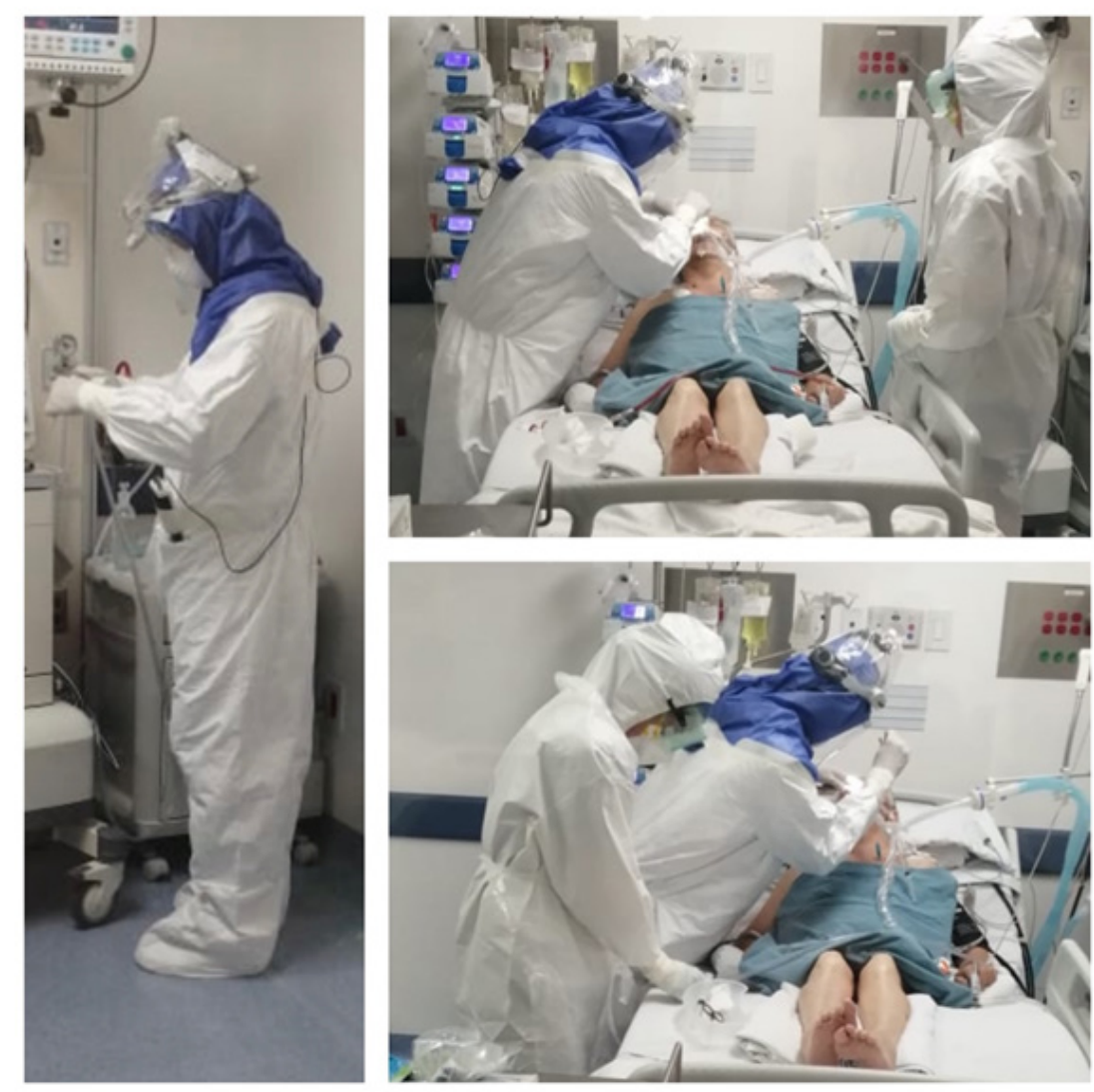

Figure 1: Management and control of posterior epistaxis in a patient with SARS CoV2.

\section{Results}

Series of 25 patients with laboratory-confirmed SARS-CoV 2 infection between March 1, 2020 and June 1, 2021, who presented epistaxis. All these patients were hospitalized, they were also on anticoagulation (Table 1) Posterior, anterior and oropharyngeal epistaxis occurred in both sexes, in patients with comorbidities (Diabetes mellitus, hypertension, obesity) without statistical difference. In those patients who were with ventilatory mechanical support presented the same risk of epistaxis, however, with a small tendency to present greater anterior epistaxis, in the same way those patients who presented anterior epistaxis had higher mortality. The median days of admission to the hospital to which they presented epistaxis was 9 days, it is worth mentioning that it was not correlated with the use of anticoagulant, since $100 \%$ of patients were on anticoagulant treatment. Those patients who presented epistaxis died 12 days after the event, without being found as the cause of this outcome. In all patients, the bleeding site was identified, controlled (Figure 2) with cauterization with silver nitrate (Silver nitrate aplicators 6 In A Mixture of 75\% silver nitrate, 25\% potassium nitrate) and absorbable hemostatic material (Gelita-Cel R, Surgicel fibrillar $\mathrm{R}$, Gelfoam R and Cutanplast R). In patients with posterior epistaxis, the posterior packing was used (Epi Stax IM), and removed after 5 days. Nasal lubricants were prescribed in all patients after bleeding was controlled. All patients required suspension of anticoagulation after the bleeding event for 48 hours. 

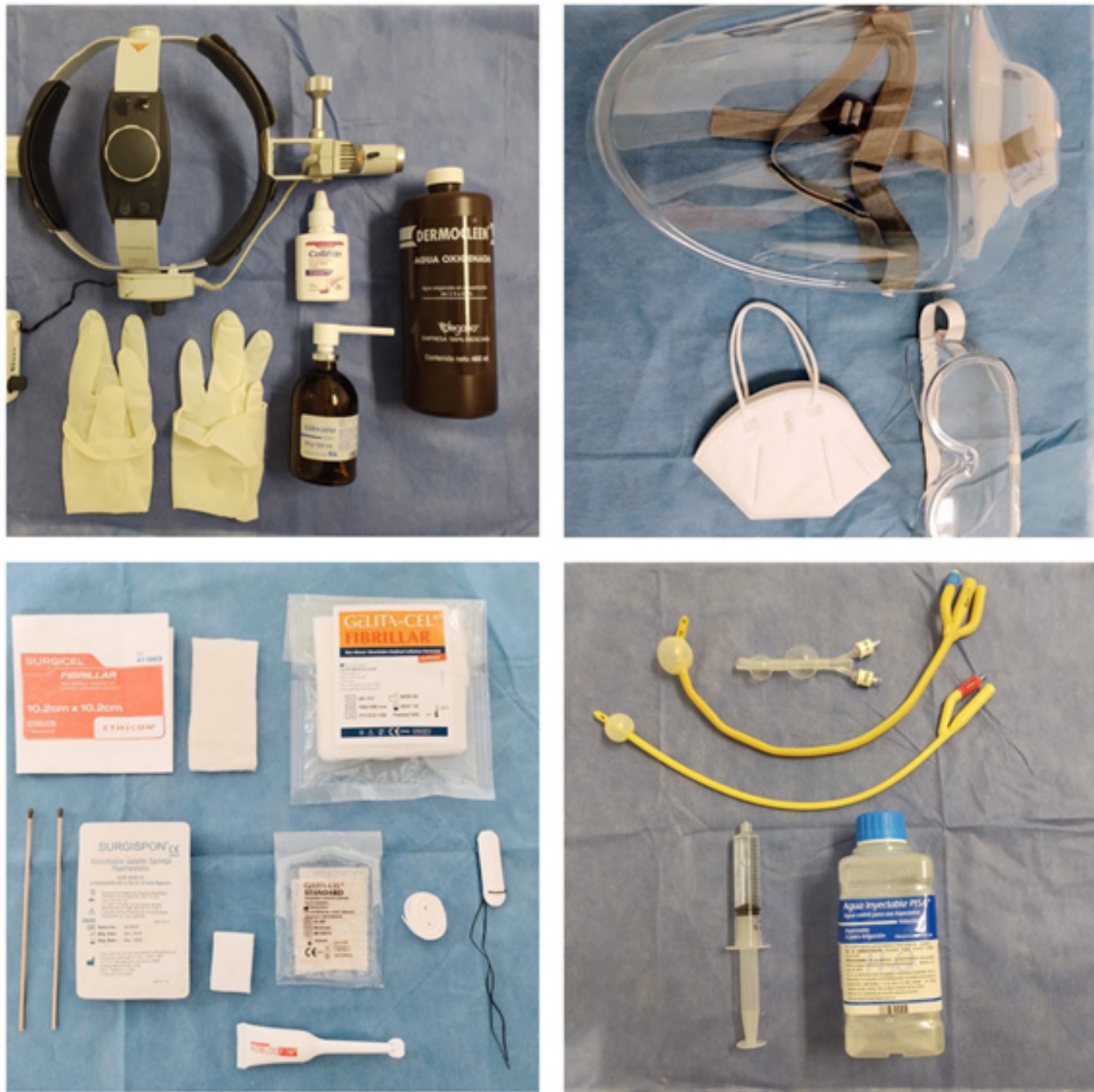

Figure 2: Equipment and material used for the treatment of bleeding in the upper airway.

Table 1: Population characteristics.

\begin{tabular}{|l|l|l|l|}
\hline \multicolumn{1}{|c|}{ Variable } & \multicolumn{1}{c|}{$\begin{array}{c}\text { Posterior Epistaxis and oro- } \\
\text { pharynx }\end{array}$} & Anterior epistaxis & Statistical value \\
\hline Sex & 4 & 11 & \\
Men & 2 & 9 & 0.545 \\
Woman & & 9 & \\
\hline $\begin{array}{l}\text { Ventilation } \\
\text { ventilatory mechanical support } \\
\text { Others }\end{array}$ & 6 & 10 & 0.051 \\
\hline $\begin{array}{l}\text { Dead } \\
\text { Yes }\end{array}$ & 6 & 9 & \\
No & 0 & 10 & 0.051 \\
\hline $\begin{array}{l}\text { Hypertension } \\
\text { Yes }\end{array}$ & 3 & 7 & \\
No & 3 & 12 & 0.653 \\
\hline $\begin{array}{l}\text { Diabetes } \\
\text { Yes }\end{array}$ & 1 & 2 & \\
No & 5 & 17 & 0.574 \\
\hline Obesity & 6 & 16 & \\
Yes & 0 & 2 & 0.554 \\
No & & & \\
\hline
\end{tabular}




\section{Discussion}

In our case series, all patients presented high prevalence of oxygen therapy in the treatment of COVID-19 pneumonia might have been the predominant cause of the high incidence of nosebleeds in this population. Finding no difference in the type of respiratory support. The use of anticoagulant drugs maybe explain the epistaxis. All of our patients were receiving LMWH for either thromboprophylaxis or therapy according to their individual risk factors and local policy. This must be taken into account because LMWH is commonly used for both in patients with low mobility and patients with COVID-19. we have a limited sample size, more observational studies are required. The use of cauterization with silver nitrate and absorbable hemostatic material may be effective for the control of upper airway bleeding.

\section{Conclusion}

Upper respiratory tract bleeding must be controlled quickly and by expert medical personnel to avoid major comorbidities in patients hospitalized for SARS CoV-2. No different methods are required to control bleeding, than use of personal protective equipment and following the international recommended measures against aerolization. Although the visualization is limited, in the hands of experts it is possible to carry out the management of the bleeding quickly and efficiently and without the need for endoscopic vision. The use of absorbable hemostatic material is preferred to avoid re-instrumentation of the upper airway that could lead to new bleeding events.

\section{Acknowledgments}

None.

\section{Funding}

None.

\section{Conflicts of Interest}

Author declares that there is no conflict of interest.

\section{References}

1. Khanal R, Oli S, Lawal H, et al. Nasopharyngeal Swab for COVID-19 Test Necessitating Mechanical Ventilation and Tracheostomy. Cureus. 2021;13(3):e13908.

2. Metlay JP, Waterer GW, Long AC, et al. Diagnosis and treatment of adults with community-acquired pneumonia. An official clinical practice guideline of the American Thoracic Society and Infectious Diseases Society of America. Am J Respir Crit Care Med. 2019;200:e45-e67.

3. Kristensen MS, McGuire B. Managing and securing the bleeding upper airway: a narrative review. Can J Anaesth. 2020;67(1):128-140.

4. Ellis MP, Hall A, Hardman J, et al. Epistaxis 2016: National audit of management. The Journal of Laryngology \& Otology. 2017;131(12):11311141.

5. Byard R. Lethal Epistaxis. J Forensic Sci. 2016.

6. Corr MJ, Tikka T, Douglas CM, et al. One-year all-cause mortality for 338 patients admitted with epistaxis in a large tertiary ENT centre. $J$ Laryngol Otol. 2019;133:487-493.

7. D’Aguanno V, Ralli M, Greco A, et al. Clinical Recommendations for Epistaxis Management During the COVID-19 Pandemic. Otolaryngol Head Neck Surg. 2020;163(1):75-77.

8. Lee DH, Kim S, Kim JS, et al. Emergent procedures for oropharyngeal bleeding during the COVID-19 pandemic: Protection of medical staff. Am J Otolaryngol. 2020;41(5):102583. 